\section{Ototoxicidade da cisplatina e otoproteção pelo extrato de ginkgo biloba às células ciliadas externas: estudo anatômico e eletrofisiológico}

\author{
Miguel Angelo Hyppolito', JoséAntônio A.de \\ Oliveira2, Maria Rossato3, Francisco Holanda
}

\section{Cisplatin ototoxycity and otoprotector to cilliated cells by ginkgo biloba extract: anatomic and eletrophisiologic study}

Palavras-chave: ototoxicidade, cisplatina, otoproteção, ginkgo biloba.

Key words: cisplatin, otoprotective agents, ginkgo biloba.
A Cisplatina é uma potente droga antineoplásica, largamente utilizada para o tratamento do câncer, tanto em adultos quanto em crianças. Dentre seus efeitos colaterais, a ototoxicidade se apresenta como um dos mais importantes e leva à perda auditiva irreversível, bilateral, para as altas freqüências (4KHz\#8K Hz). Estudos têm tentado identificar drogas que, associadas à cisplatina possam atuar como otoprotetores. Sabe-se que o mecanismo da ototoxicidade pela cisplatina está relacionado a alterações nos mecanismos antioxidantes das células ciliadas, principalmente as células ciliadas externas da cóclea. Objetivo: Nossa proposta foi de avaliar através de emissões otoacústicas, por produtos de distorção (EOAPD) e por microscopia eletrônica de superfície (ME), a ação do extrato de ginkgo biloba (EGB 761), que tem conhecida ação antioxidante, como possível otoprotetor, utilizando como modelo experimental cobaias albinas. Forma de estudo: Experimental. Material e método: Observamos EOAPD presentes pré e pós tratamento no grupo EGB (100 mg/Kg/dia via oral) e 90 minutos após cisplatina ( $80 \mathrm{mg} / \mathrm{Kg} /$ dia via intraperitoneal) por 8 dias. Resultado: Houve também manutenção da arquitetura ciliar nas células ciliadas externas em todas as espiras da cóclea, enquanto que no grupo tratado somente com cisplatina (80 mg/ $\mathrm{Kg}$ /dia via intraperitoneal) por 8 dias, houve desaparecimento das EOAPD pós tratamento, com desaparecimento dos cilios das células ciliadas externas e distorção na arquitetura dos cílios remanescentes à ME. Conclusão: Concluímos que a EGB, por sua ação antioxidante, atua como fator otoprotetor à ototoxicidade pela cisplatina, devendo ser testada tal ação na prática clínica em pacientes que utilizam a cisplatina, pois o uso do EGB está extremamente difundido no tratamento de diferentes doenças. isplatin is an antineoplastic drug for cancer treatment in children and adults. The side effects of cisplatin ototoxycity are important with irreversible auditory and bilateral damage to high frequencies $(4 \mathrm{kHz}-8 \mathrm{kHz}$ ). Reports recognize some drugs that are associated with cisplatin to obtain an otoprotector effect. The ototoxycity mechanisms of cisplatin are related to injury of conduct the hair cell oxidation mechanism, with particular injury to outer hair cells. Aim: We intend to studies using otoacoustic emissions - distortion products (DPOEA) and scanning microscopy to verify the action of ginkgo biloba (GBE-761) that has well known antoxidizing characteristics, that can function like otoprotector effects. Study design: Experimental. Material and method: We use an experimental guinea pig model. We found DPOEA positive before and after treatment in the GBE group (100 mg/ Kg/ day - oral) and after 90 minutes cisplatin ( $8,0 \mathrm{mg} / \mathrm{Kg} /$ day - intraperitoneal - 8 consecutive days). Results: The normal cilium architecture of outer hair cells was supported in all cochlear spirals and in the group treated only with cisplatin ( $8,0 \mathrm{mg} / \mathrm{Kg} /$ day - intraperitoneal - 8 consecutive days), the DPOEA was not present and strong injury to cilium of outer hair cells showed cilium disorders upon scanning microscopy. Conclusion: We conclude that GBE has a potential otoprotector effect against cisplatin ototoxycity and could be used in clinical trials.

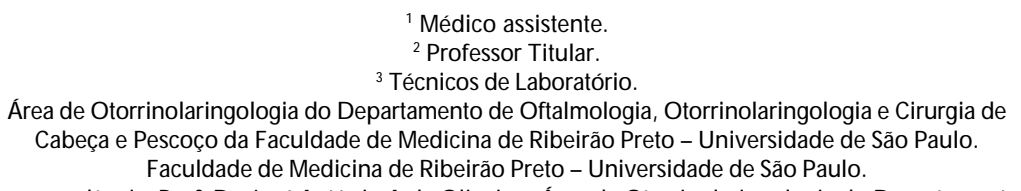

Faculdade de Medicina de Ribeirão Preto - Universidade de São Paulo.

Endereço para Correspondência: Prof. Dr. José Antônio A.de Oliveira - Área de Otorrinolaringologia do Departamento de Oftalmologia, Otorrinolaringologia e Cirurgia de Cabeça e Pescoço da Faculdade de Medicina de Ribeirão Preto - Universidade de São Paulo - Campus USP. Avenida Bandeirantes, 3900 Ribeirão Preto SP 14049-900

Tel (0xx16) 602-2863 - Fax (0xx16) 602-2860

Artigo recebido em 13 de maio de 2003. Artigo aceito em 05 de junho de 2003. 


\section{INTRODUÇÃO}

A cisplatina é uma potente droga antineoplásica utilizada em larga escala tanto em adultos quanto em crianças na terapia do câncer avançado. Muitos de seus efeitos colaterais são irreversíveis e podem ser monitorados clinicamente, mas freqüentemente não podem ser evitados. Diversos estudos têm sido conduzidos no intuito de descobrir drogas que, associadas a cisplatina, possam evitar os efeitos indesejáveis desta. ${ }^{1-9}$

A toxicidade da cisplatina tem sido verificada tanto em nível renal, como do sistema nervoso central ou periférico, toxicidade gastrointestinal e da medula óssea e, assim como outras classes de medicamentos, causa lesões ao órgão de Corti, sendo considerada uma droga ototóxica. ${ }^{1,2,4,7,8}$

Estudos anatômicos demonstram que a cisplatina provoca danos tanto com doses agudas elevadas como com doses cumulativas, principalmente com o comprometimento de células ciliadas externas, havendo lesão inicial nas células da espira basal da cóclea. Alguns autores sugerem a ocorrência de um bloqueio na transdução dos canais de cálcio destas células, outros estudos mostram lesões às células ciliadas internas, às células suportes e "stria vascularis", bem como 0 gânglio espiral. ${ }^{10-17}$

A cisplatina causa uma perda auditiva bilateral e irreversível em humanos, com zumbido associado e comprometendo as altas freqüências $(4.000 \mathrm{~Hz}$ a 8.000 $\mathrm{Hz}) ., 2,2,8,18$

As formas de se estudar os efeitos da toxicidade coclear têm se dirigido às alterações eletrofisiológicas detectadas pelo Potencial Evocado de Tronco Cerebral (BERA) e Potencial Endococlear. 2,4,5,15,16,19-21

As emissões otoacústicas, detectadas por Kemp (1971) no conduto auditivo externo de humanos, medem o "feedback" de energia biomecânica na contração das células ciliadas externas, que amplifica o pico da "onda viajante" na membrana basilar e têm aplicação clínica na avaliação da integridade auditiva. . $^{1621-25}$

Como a cisplatina atua inicialmente causando lesões nas células ciliadas externas, as emissões otoacústicas seriam um método de avaliação simples e rápido de tais danos, podendo inclusive participar na monitorização de pacientes submetidos a drogas consideradas ototóxicas. ${ }^{16,21-26}$

Estudos têm demonstrado um possível mecanismo antioxidante para a ototoxicidade e nefrotoxicidade geradas pela cisplatina, pois as vias de detoxicação nos dois tecidos são semelhantes. Autores mostram que os níveis de glutation e a atividade de enzimas antioxidantes como superóxido dismutase, catalase, GSH peroxidase e GSH redutase estão reduzidas nestes tecidos, levando a peroxidação lipídica, instalando assim, a toxicidade celular. 10,27-34 $^{2}$

Diversas drogas têm sido testadas com o objetivo de oto proteção como o tiossulfato de sódio, o dietilcarbamato, o ACTH e derivados, 0 ácido 4-metilthiobenzóico, o ácido lipóico, o glutation e seus ésteres, metionina, procaína, hormônio estimulador alfa-melanocítico (melatonina), antioxidantes como a ginkgo biloba e fosfomicina e compostos sulfurados. $3.5,6,9,3,35-47$

Trabalho recente de tese de mestrado da FMRP USP mostrou que baixas doses de gentamicina levam a um efeito protetor estrutural para as células ciliadas externas, que mantém suas características normais à microscopia eletrônica de varredura quando se aplicam, posteriormente, doses elevadas desta droga, sugerindo assim, que existe um mecanismo de autodefesa - adaptação intra-coclear às agressões induzidas pelas drogas o totóxicas. ${ }^{48-51}$

0 objetivo da investigação é verificar se existe o efeito oto protetor de drogas que já são utilizadas na prática clínica com outras finalidades como o extrato de ginkgo biloba, aos danos cocleares causados pela cisplatina, utilizando doses de cisplatina sabidamente ototóxicas, lesivas às células ciliadas externas, avaliando-se as alterações anatômicas através da microscopia eletrônica de superfície, e avaliação funcional por emissões otoacústicas - produtos de distorção.

\section{MATERIAL E MÉTODO}

Elegemos como animal experimental cobaias albinas, pelo fácil manejo, pela facilidade de dissecção coclear e manipulação, facilidade para vias de infusão para as drogas anestésicas e drogas em experimentação, pela via intraperitoneal ou intramuscular, bem como, porque quando comparada ao rato, por exemplo, é mais sensível aos efeitos da cisplatina, podendo-se manter adequados cuidados, seguindo as normas de cuidados para com animais experimentais. ${ }^{11,12,14,37,52-54}$

Os animais foram divididos em 4 grupos de estudo:

Grupo 1: 4 animais- 8 cócleas- cisplatina 8,0 mg/ $\mathrm{Kg} /$ dia (IP) por 8 dias.

Grupo 2: 6 animais - 12 cócleas - Ginkgo biloba $100 \mathrm{mg} / \mathrm{Kg}$ via oral e 90 minutos após, cisplatina $8,0 \mathrm{mg} /$ $\mathrm{Kg} / \mathrm{dia}$ (via intraperitoneal) por 8 dias.

Grupo 3: 6 animais - 12 cócleas - Ginkgo biloba $100 \mathrm{mg} / \mathrm{Kg}$ via oral por 10 dias e a pós Ginkgo biloba 100 $\mathrm{mg} / \mathrm{Kg}$ via oral e 90 minutos após, cisplatina $8,0 \mathrm{mg} / \mathrm{Kg} /$ dia (via intraperitoneal) por 8 dias.

Grupo 4: 3 animais - 6 cócleas- Ginkgo biloba 100 $\mathrm{mg} / \mathrm{Kg}$ via oral por 8 dias.

Drogas utilizadas:

1. Cisplatina $(8,0 \mathrm{mg} / \mathrm{ml})$ - Laboratório Farmacêutico Eurofarma

$8,0 \mathrm{mg} /$ dia via intraperitoneal

2. Xilazina (Coopazine ${ }^{\circledR}$, Rompun $\left.{ }^{\circledR}\right) 2 \mathrm{~g} / 100 \mathrm{ml}(6,5 \mathrm{mg} / \mathrm{Kg}$ )

3. Cloridrato de Cetamina (Ketamin $\left.{ }^{\circledR}\right) 50 \mathrm{mg} / \mathrm{ml}$ (65mg/ $\mathrm{Kg}$ ) 
4. Extrato de ginkgo biloba (EGB 761) - Laboratório Farmacêutico - ByK Química 40 mg/ml $(100 \mathrm{mg} / \mathrm{Kg} /$ dia)

1. Emissões O toacústicas por Produto de Distorção EUIPAMENTO: ILO 92 CAE System O todynamics LTD. 2. Microscopia Eletrônica de Varredura Microscópio Jeol - JSM 5200.

\section{Emissões Otoacústicas por produtos de distorção:}

As cobaias foram anestesiadas com Coopazine ${ }^{\circledR} \mathrm{e}$ Ketamin ${ }^{\circledR}$ para serem submetidas ao teste. Antes da realização do teste foram submetidas a otoscopia manual para avaliação do conduto auditivo externo e membrana timpânica, sendo descartadas as cobaias que apresentaram sinais de otites e as que apresentaram cerume que não pode ser removido.

0 teste de emissões otoacústicas por produtos de distorção foi realizado pré tratamento e momentos antes dos animais serem sacrificados, seguindo a relação $2 \mathrm{~F} 1$ - F2 com relação $F 1: F 2=1,22$, resolução 1 ponto por oitava.

\section{Microscopia Eletrônica de Varredura ${ }^{11,33,49,55}$}

As cobaias foram sacrificadas em tempo programado após administração das drogas via intraperitoneal e então sacrificadas após anestesia com éter, tendo sido decapitadas e suas cócleas removidas da bula.

Com dissecção microscópica as cócleas foram perfundidas com solução de fixação com glutaraldeído 3\% a 4 ํㅡㄴ sius e mantidas na solução por 24 horas para a fixação. Os passos seguintes foram realizados no Laboratório de Microscopia Eletrônica do Departamento de Morfologia da FMRP-USP.

Através da janela redonda foi injetado para fixação uma solução de glutaraldeído a $3 \%$ em tampão fosfato 0,1 $\mathrm{M}, \mathrm{pH}=7,4$, por 4 horas a 4은 Celsius, lavadas 3 vezes por 5 minutos com o mesmo tampão, após o que foram fixadas com tetróxido de Ósmio 1\% por 2 horas a 40 Celsius e submetidos a uma desidratação à temperatura ambiente em uma bateria crescente de etanol $(50 \%, 70 \%, 90 \%$ e $95 \%$ uma vez por 10 minutos em cada concentração) e etanol absoluto 3 vezes por 15 minutos. Terminada a desidratação, seguiu-se para a secagem pelo método do ponto crítico em $\mathrm{CO}_{2}$, onde o material ficou desprovido de água. Após ser fixado em porta espécime adequado, o material foi revestido em câmara de vácua com vapores de ouro e examinados em microscópio eletrônico de varredura.

Foi utilizado o Microscópio Eletrônico JEOL SCANNING MICRO SCOPE - JSM 5200.

O s resultados obtidos da microscopia eletrônica de varredura, após fotografados, foram analisados através de cócleogramas (contagem do número de células ciliadas externas da espira basal da cóclea em determinado campo fotográfico) e avaliadas estatisticamente pelo t-test, utilizando o programa estatístico - Primer of Biostatistics (Stanton A. Glant).

\section{RESULTADOS}

Com relação à dosagem de Cisplatina utilizada, em trabalho anterior (dados não publicados), utilizamos a dosagem de $1,5 \mathrm{mg} / \mathrm{Kg} /$ dia por 18 dias e não observamos alterações anatômicas nas células ciliadas externas na espira basal da cóclea de cobaias albinas tratadas com Cisplatina, bem como não ocorreram alterações ás emissoes otoacústicas por produtos de distorção pré e pós tratamento. (Figura 1)

A avaliação do estado funcional das células ciliadas externas, estudadas pelas emissões otoacústicas por produtos de distorção, mostraram redução na amplitude nas diferentes freqüências testadas a partir de $2 \mathrm{kHz}$, sendo as respostas identificadas na zona de ruído no grupo tratado com cisplatina $8,0 \mathrm{mg} / \mathrm{Kg} /$ dia por 7 dias. (Figura 2)

Q uanto às emissões otoacústicas no grupo 2, tratados com ginkgo biloba previamente a cisplatina, os resultados estão representados na Figura 3.

As emissões otoacústicas por produtos de distorção avaliam o estado funcional das células ciliadas externas na espira basal da cóclea, no entanto, a avaliação é qualitativa. Foram consideradas as emissões otoacústicas por produtos de distorção como presentes e ausentes. Nos grupos 2 e 3 tratados com ginkgo biloba as emissões otoacústicas por produtos de distorção estiveram presentes em todas as orelhas testadas.

Comparando-se os grupos 2 e 3, observamos que 0 traçado das emissões otoacústicas por produtos de distorção mostraram-se presentes e em níveis de intensidade maiores $(\mathrm{dB})$, mas os dados não puderam ser testados estatisticamente.

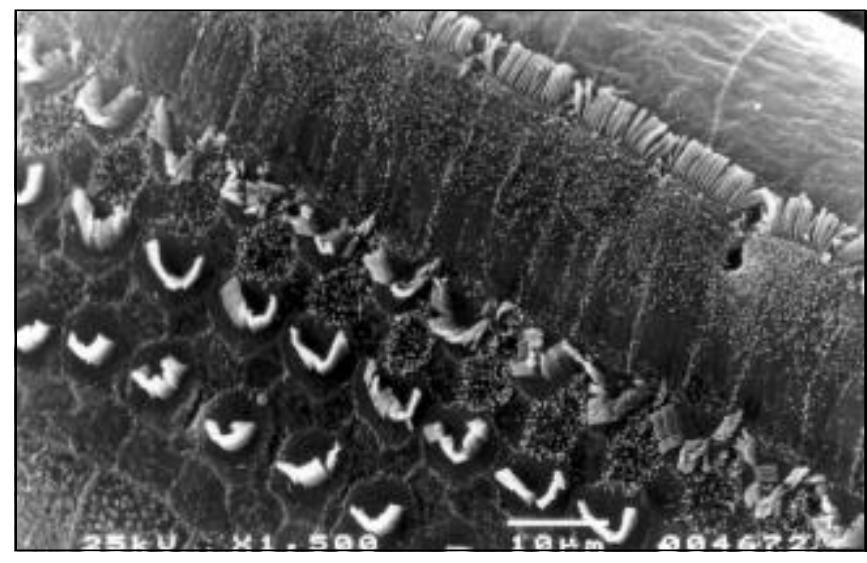

Figura 1. Foto de Microscopia Eletrônica de Varredura de uma das cobaias tratadas com Cisplatina 1,5 mg/Kg/dia por 18 dias - pós tratamento (no dia do sacrifício). 


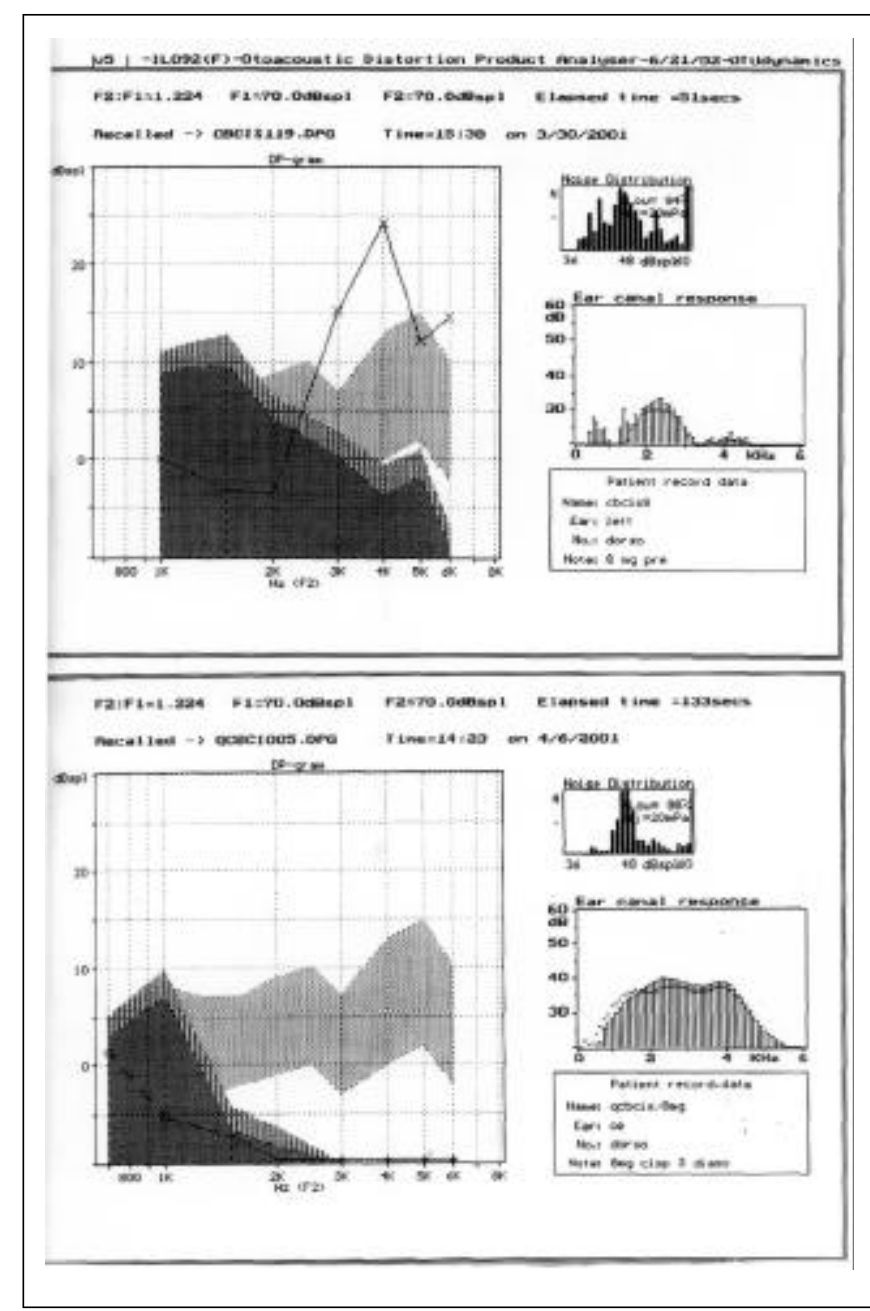

Figura 2. emissões otoacústicas - produto de distorção de uma das cobaias tratadas com cisplatina $8,0 \mathrm{mg} / \mathrm{Kg} / \mathrm{dia}$ por 8 dias -pré e pós tratamento (no dia do sacrifício).

Entre os grupos 1 e 2 e 1 e 3 as emissões otoacústicas por produtos de distorção estiveram ausentes em todas as orelhas do grupo $1(n=8)$, com diferença estatisticamente significante. (Fisher exact test: $p=0,003$ )

$Q$ uanto à avaliação anatômica, no grupo tratado com cisplatina isolada $(8,0 \mathrm{mg} / \mathrm{kg} / \mathrm{dia}$ ) houve lesão com ausência de cílios nas 3 fileiras de células ciliadas externas a nível da espira basal, seguida da espira 2 e espira 3. As alterações mais evidentes foram na espira basal, mas observamos também distorção ciliar com desarranjo no padrão em " $\mathrm{v}$ " (ou "W"), com cílios dobrados ou ausência parcial de um dos "braços"do " $v$ " (Figura 4). Em nível das células ciliadas internas também observamos alterações ciliares, com cílios presentes, mas desarranjados.

No grupo tratado previamente com ginkgo biloba (grupo 2), notamos a presença de todas a células ciliadas externas na espira basal sem a distorção dos cílios tanto nas

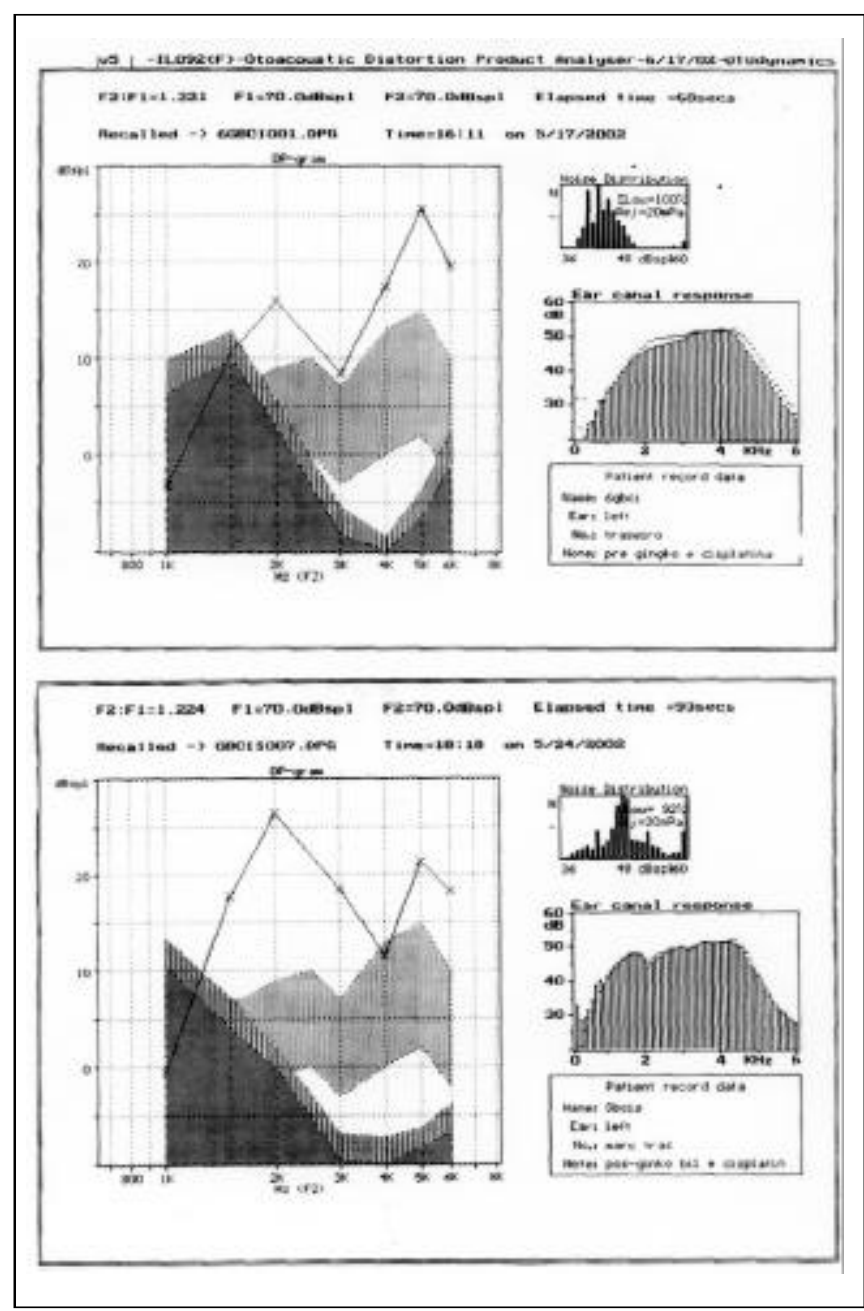

Figura 3. emissões otoacústicas - produto de distorção de uma das cobaias tratadas com ginkgo biloba $100 \mathrm{mg} / \mathrm{Kg} /$ dia 90 minutos antes da cisplatina $8,0 \mathrm{mg} / \mathrm{Kg} / \mathrm{dia}$ por 8 dias - pré e pós tratamento (no dia do sacrifício).

células ciliadas externas quanto nas internas. Nas espiras 2 e 3 ocorreu rara ausência de cílios nas fileiras 1 e 2 das células ciliadas externas, sem distorção ciliar e sem alterações nas células ciliadas internas. (Figura 5).

Selecionamos um quarto grupo com 3 cobaias onde utilizamos somente 0 extrato de ginkgo biloba na dosagem de $100 \mathrm{mg} / \mathrm{Kg} /$ dia por 8 dias, e observamos que as emissões otoacústicas mantiveram-se presentes neste período e a microscopia eletrônica de superfície mostrou a presença de células ciliadas externas em todas as espiras da cóclea.

Q uanto à análise estatística dos dados colhidos através do cócleograma da epira basal das cócleas estudadas entre os grupos 2 e 3 não houve diferença estatisticamente significante (t-test: $p=0,591$ ).

Q uando os dados de cócleograma dos grupos 1 e 2 e grupos 1 e 3 foram analisados estatisticamente encontramos diferenças significantes estatisticamente (t-test: $p<0,001$ ). 


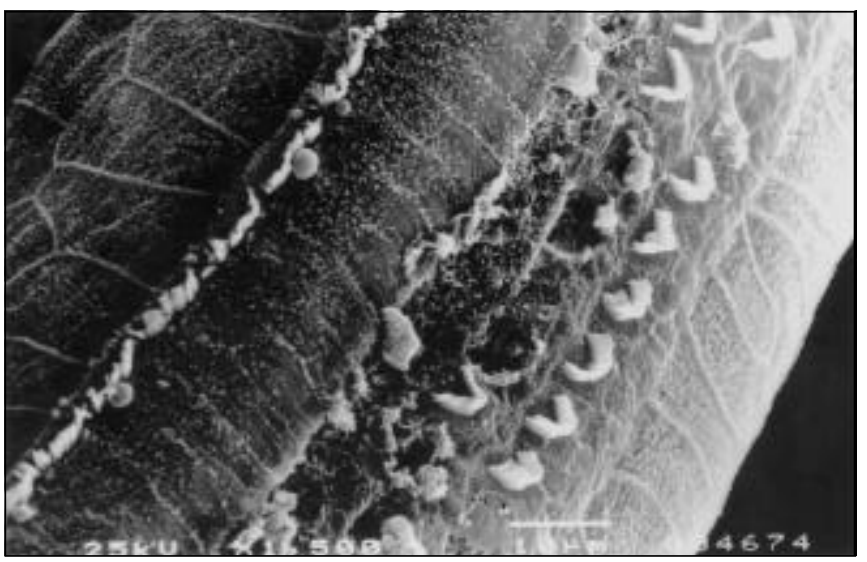

Figura 4. Microscopia Eletrônica de superfície da cóclea de cobaia tratada com cisplatina $8,0 \mathrm{mg} / \mathrm{Kg} / \mathrm{dia} 8$ dias. Alteração nas células ciliadas externas, 3 fileiras de cilios com desarranjo dos cilios remanescentes e distorção ciliar nas células ciliadas internas (espira basal).

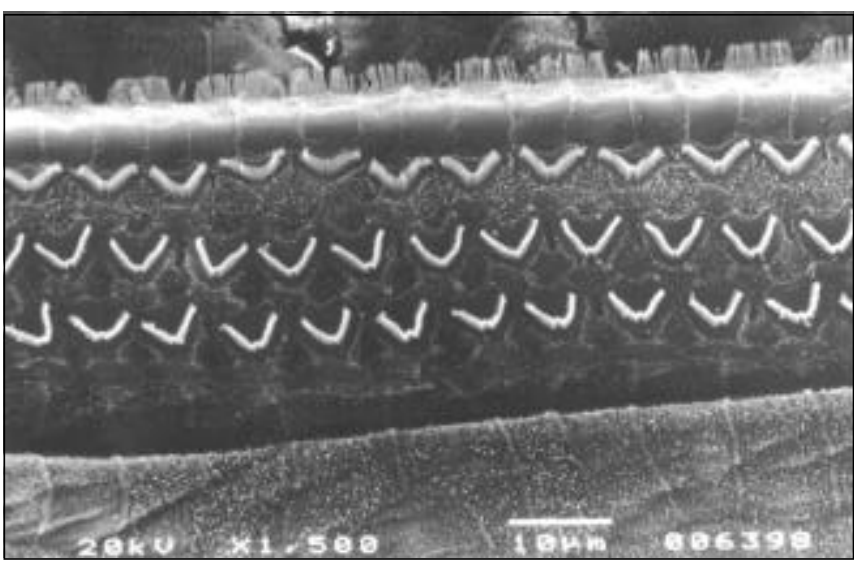

Figura 5. Microscopia Eletrônica de superfície da cóclea de cobaia tratada com extrato de ginkgo biloba $100 \mathrm{mg} /$ dia e cisplatina 8,0 $\mathrm{mg} / \mathrm{Kg} /$ dia 8 dias. Padrão normal na espira basal da cóclea com organização ciliar e padrão normal dos cílios das células ciliadas internas.

\section{DISCUSSÃO}

Sabendo-se que a Cisplatina leva a ototoxicidade por lesão das células ciliadas externas e que as emissões otoacústicas avaliam o estado de contração das células ciliadas externas, sendo este um método simples e representativo do estado funcional destas células, as emissões otoacústicas por produto de distorção foram utilizadas neste estudo.

Até recentemente, a maioria dos trabalhos sobre ototoxicidade pela cisplatina avaliavam as alterações funcionais da cóclea através dos potenciais de ação, somação e microfônico coclear ou através da audiometria de tronco cerebral (BERA). Sokalingam et al. (2000) verificaram que o registro das emissões otoacústicas, transientes e por produto de distorção é um méto do sensível de avaliação do estado funcional das células ciliadas externas, e que a cobaia albina é 0 animal mais sensível em termos de ototoxicidade pela cisplatina, sendo que nas doses de 10 a $12 \mathrm{mg} / \mathrm{kg} / \mathrm{dia}$, em 3 dias já existe alteração nas emissões otoacústicas por produtos de distorção e lesão das células ciliadas externas, com depressão na amplitude dos produtos de distorção maior do que nas emissões otoacústicas transientes. ${ }^{16,21,23-26,54}$

Com relação à dose da cisplatina escolhida para o estudo, diversos trabalhos da literatura apontam as dosagens, via de administração e tempo de uso ideais para o estudo dos efeitos de oto toxicidade pela cisp latina. Cardinal et al. (2000) mostram que nas doses de 1,5 a $2,0 \mathrm{mg} / \mathrm{Kg} / \mathrm{dia}$ de cisplatina intraperitoneal, em co baias al binas oco rre $60 \%$ a $65 \%$ de perda celular em 8 dias, evidenciada na espira basal da cóclea. ${ }^{11,17,18,46,51}$

Nas doses de 8,0 a 15,0 mg/Kg intraperitoneal, dose única, já existem alterações nas emissões otoacústicas por produtos de distorção e na audiometria de tronco cerebral. ${ }^{11,54}$

Escolhemos a dose de $80 \mathrm{mg} / \mathrm{Kg} /$ dia por 8 dias, pois seguramente encontraríamos lesões de mais de $65 \%$ das células ciliadas externas na espira basal, com alterações evidentes nas emissões otoacústicas por produto de distorção e à microsco pia eletrônica. ${ }^{11}$

No grupo em estudo onde utilizamos $80 \mathrm{mg} / \mathrm{Kg} / \mathrm{dia}$, em 3 a 7 dias notamos alterações com lesão celular às três fileiras de células ciliadas externas e algumas alterações ciliares na fileira de células ciliadas internas.

Com relação aos mecanismos envolvidos na ototoxicidade pela cisplatina, diferentes estudos têm tentado diferenciar tais mecanismos, para se poder atuar com mais precisão e eficácia com o uso de agentes otoprotetores.

Ravi et al. (1995), estudando os efeitos da cisplatina sobre os mecanismos antioxidantes na cóclea através da medida nos níveis de glutation, glutation oxidado e malonil dialdeído, bem como atividade de enzimas antioxidantes, como superóxido dismutase, catalase, glutation peroxidase e glutation redutase, verificaram que a atividade da superóxido dismutase e catalase e os níveis de malonildialdeído estavam significantemente aumentados, e, as atividades da glutation peroxidase e glutation redutase estavam significativamente reduzidas nas cócleas sob ação da cisplatina. ${ }^{27}$

Trabalhos recentes mostram que tal mecanismo pode explicar os efeitos ototóxicos da cisplatina. .0,33,40,50 $^{2}$

Smoorenburg et al. (1999) mostram que as alterações nos sistemas antioxidantes das células ciliadas externas são importantes, e que diferentes agentes otoprotetores testados até o momento atuam prevenindo a interação da platina com a superóxido dismutase, impedem a formação de radicais livres, deslocam a platina do seu sítio tóxico e podem interagir diretamente com a cisplatina, no entanto, estes dois últimos mecanismos podem interferir na potência antineoplásica da cisplatina. ${ }^{32,50}$ 
Huang et al. (2000) mostram a lesão coclear com apoptose das células ciliadas externas submetidas a stress oxidativo. A cisplatina leva à formação de espécies de oxigênio reativo e radicais livres intracelulares, que interagem com os fosfolípides de membrana das células ciliadas externas, levando à peroxidação aldeído lipídica (4hidroxinonenal) que é um conhecido mediador de apoptose para as células ciliadas externas e neurônios auditivos. Assim, propõem que a otoproteção deve atuar nos mecanismos de anti-oxidação, podendo-se atuar em três pontos básicos: 1) prevenção da formação de oxigênio reativo, 2) neutralização de produtos tóxicos da peroxidação lipídica e 3) bloqueio às substâncias que causam dano à membrana das células sensoriais, bloqueando os mediadores de apoptose. ${ }^{33,34}$

Diferentes substâncias vêm sendo testadas no intuito de otoproteção a cisplatina, como: dietilcarbamato, ácido 4metiltiobenzóico, ebselen, melatonina, ácido lipóico 9,40,42,43,45, acetil cisteína ${ }^{38}$, fosfomicina ${ }^{47}$, neurotrofinas ${ }^{39}$, análogos do ACTH. $20,41,44$

Muitas destas substâncias têm efeitos colaterais in vivo e não se sabe sobre sua possível atuação bloqueando os efeitos antineoplásicos da cisplatina.

Fukaya et al. (1999) testaram os efeitos do extrato seco de ginkgo biloba (egb 761) sobre a ototoxicidade pela cisplatina como sendo um possível agente otoprotetor. Em tal estudo foram utilizados ratos albinos, a dosagem de cisplatina utilizada foi de $1,5 \mathrm{mg} / \mathrm{Kg} /$ dia durante 10 dias, com medidas fisiológicas dos potenciais de ação e estudo histológico. Observaram que a ginkgo biloba protege as células ciliadas externas. ${ }^{46}$

A Ginkgo biloba apresenta propriedades antioxidantes, atua diminuindo a peroxidação lipídica, aumentando os níveis de catalase, superóxido dismutase e glutation. No plasma, também atua formando complexos com fosfolípides, aumentando sua atividade antioxidante. Em nível intracelular, atua em mitocôndrias, na fosforilação oxidativa do RNA mitocondrial e a nível do Complexo de Golgi, auxiliando no mecanismo de reparo do DNA com melhora do "status antioxidante" celular, com aumento dos níveis de glutation intracelular e diminuição na incorporação de 3H-timidina. ${ }^{56-62}$

Nossa proposta foi de tentar confirmar tais achados, mas utilizando como animal experimental a cobaia albina, que tem se mostrado muito mais sensível aos efeitos ototóxicos da cisplatina (quando comparado ao rato albino) e utilizar doses elevadas de cisplatina, que são seguramente lesivas para as células ciliadas externas. ${ }^{16,46}$

Como Fukaya et al., observamos que existe um efeito protetor da ginkgo biloba para as células ciliadas externas, não encontramos alterações às emissões otoacústicas por produtos de distorção após 8 dias de uso da cisplatina ( 80 $\mathrm{mg} / \mathrm{Kg} / \mathrm{dia}$ ), quando da associação com a ginkgo biloba, não encontramos lesão celular à células ciliadas externas a microscopia eletrônica de varredura, na espira basal da cóclea; nas espiras 2 e 3 encontramos a ausência de 1 ou 2 células em metade das cócleas analisadas.
Considerando os mecanismos de ototoxicidade pela cisplatina, relacionados aos mecanismos de antioxidação celular e formação de radicais livres, e, sendo que o extrato de ginkgo biloba leva à redução da peroxidação lipídica, com limpeza de superóxidos, impedindo a formação de radicais livres, além de ser um medicamento largamente utilizado em humanos e com efeitos colaterais considerados pouco significativos e as evidências de otoproteção à cisplatina, existe a possibilidade de uso concomitante da ginkgo biloba e cisplatina para tentar-se minimizar os efeitos ototóxicos desta, tanto em adultos quanto em crianças.

\section{CONCLUSÕES}

1. 0 extrato seco de ginkgo biloba (egb 761) tem um efeito protetor para as células ciliada externas, em cócleas de cobaia albina contra a agressão pela cisplatina, provavelmente por seu efeito modulador dos sistemas de defesa anti-oxidantes destas células, impedindo assim a formação de radicais livres celulares ou favorecendo sua eliminação pela célula exposta ao stress oxidativo, conforme acredita-se ser o mecanismo de ação da ginkgo biloba.

2. Por ser uma droga largamente utilizada na prática clínica e por não serem descritos efeitos colaterais importantes, bem como não existirem descrições sobre interações com outras drogas, a Ginkgo Biloba poderá ser estudada em seres humanos como potencial agente otoprotetor aos efeitos de drogas o totóxicas como a Cisplatina.

\section{REFERÊNCIASBIBUOGRÁFICAS}

1. Laurell G, Engström B, Bagger-Sjöback D. Ototoxicity of Cisplatin. Int J Androl 1987;10:359-62.

2. Powis GD, Hacker MP. The Toxicity of Anticancer Drugs. New York: Pergamon Press; 1991. 82-105.

3. Rybak LP, Radhika R, Somani SM. Mechanism of Protection by Diethyldithiocarbamate against Cisplatin Ototoxicity: Antioxidant System. Fundamental and Apllied Toxicology. 1995;26:293-300.

4. Stadnicki SW, Gleischman RW, Schaaeppi U. Ototoxicity of Cisdichlorodiammine Platinum (II) (NSC-119875): Hearing loss and Other Toxic Effects in Rhesus Monkeys. Can Chemother Ver 1975;59:467-80.

5. Stengs CHM, Klis SFL, Huizing EH, Smoorenburg GF. Protective Effects of a Neurothophic ACTH (4-9) Analog on Cisplatin O totoxicity in Relation to the Cisplatin Dose: An Electrocochleographic Study in Albino Guine Pigs. Hearing Res 1998;124:108-17.

6. Tognela S. Pharmacological Interventions To Reduce Platinuminduced Toxicity. Can Trat Ver 1990;17:139-42.

7. Towflight J, Strauss M, Lord S. Cisplatin Ototoxicity: Clinical Experience and Temporal Bone Hystopatology. Laryngoscope 1983;93:1554-9

8. Wright CG, Schaeffer SD. Inner Ear Hystophatology in patients Treated With Cisplatin. Laringoscope 1982;92:1408-13.

9. Rybak LP, Husain K, Morris C, Whitworth C, Somani S. Effect of protective agents against cisplatin ototoxicity. Am J Otol 2000;21(4):513-20.

10. Barron SE, Dainneault EA. Effect of Cisplatin on Hair Cell Morphology and Lateral Wall Na-K-ATP ase Activity. Hear Res 1987;26:131-7. 
11. Cardinaal RM, Groot JCMJ, Huizing EH, Veldman JE, Smoorenburg GF. Dose-Dependent Effect of 8-Day Cisplatin Administration Upon the Morphology of the Albino Guinea Pig Cochlea. Hearing Res 2000;144:135-46

12. Janning MH, Withworth CA, Rybak LP. Experimental Model of Cisplatin Ototoxicity in Chinchilas. Otolaryngol Head Neck Surg 1998;119(6):574-80.

13. Kohn SM, Fradis J, Zidan L, Podoshin E, Robinson \& I Nir. Cisplatin Ototoxicity in Guinea Pigs with Special Reference to Toxic Effects in the Stria Vascularis. Laringoscope 1988;8;885-71.

14. Laurell G, Engström B. The Ototoxic Effect of Cisplatin in Guinea Pigs in Relation to Dosage. Hear Res 1989;38:27-34

15. Stengs CHM, Klis SFL, Huizing EH, Smoorenburg GF. Cisplatin Ototoxicity. An Electrophysiological Dose-Effect Study in Albino Guinea Pigs. Hearin Res 1998;124:99-107.

16. Sockalingam R, Freeman S, Cherny TL, Sohmer H. Effect of highdose cisplatin on auditory brainstem responses and otoacoustic emissions in laboratory animals. Am J Otol 2000; 21(4):521-7.

17. Stengs $\mathrm{CH}$, Klis SF, Huizing EH, Smoorenburg GF. Cisplatin ototoxicity. An electrophysiological dose-effect study in albino guinea pigs. Hear Res 1998;124(1-2):99-107.

18. Saito T, Aran JM. Comparative ototoxicity of cisplatin during acute and chronic treatment. ORL J Otorhinolaryngol Relat Spec 1994;56(6):315-20.

19. Shaw NA. The Auditory Evoked Potential in the Rat - a Review. Prog Neurobiol 1988;31:19-45.

20. Stengs $\mathrm{CH}, \mathrm{Klis} \mathrm{SF}$, Huizing EH, Smoorenburg GF. Protective effects of a neurotrophic ACTH(4-9) analog on cisplatin ototoxicity in relation to the cisplatin dose: an electrocochleographic study in albino guinea pigs. Hear Res 1998;124(1-2):108-17.

21. Sie KC, Norton SJ. Changes in otoacoustic emissions and auditory brain stem response after cis-platinum exposure in gerbils. Otolaryngol Head Neck Surg 1997;116(6 Pt 1):585-92.

22. Harel N, Kakigi. A, Hirakawa H, Mount RJ, Harrison RV. The Effects of Anesthesia on Otoacoustic Emissions. Hearing Res 1997:110:25-33

23. Cevette MJ, Drew D, Webb TM, Marion MS. Cisplatin ototoxicity increased DPOAE amplitudes and magnesium deficiency. Distortion product otoacoustic emissions. J Am Acad Audiol 2000;11(6):323-

24. Allen GC, Tiu C, Koike K, Ritchey AK, Kurs-Lasky M, Wax MK. Transient-evoked otoacoustic emissions in children after cisplatin chemotherapy. Otolaryngol Head Neck Surg 1998;118(5):584-8.

25. Ozturan 0 , Jerger J, Lew $H$, Lynch GR. Monitoring of cisplatin ototoxicity by distortion-product otoacoustic emissions. Auris Nasus Larynx 1996;23:147-51.

26. Ress BD, Sridhar KS, Balkany TJ, Waxman GM, Stagner BB Lonsbury-Martin BL. Effects of cis-platinum chemotherapy on otoacoustic emissions: the development of an objective screening protocol. Third place Resident Clinical Science Award 1998. Otolaryngol Head Neck Surg 1999;121(6):693-701.

27. Ravi R, Somani SM, Rybak LP. Mechanism of Cisplatin Ototoxicity: Antioxidant System. Pharmacology \& Toxicology. 1995;76:38694.

28. McAlpine D, Johnstone BM. The Ototoxic Mechanism of Cisplatin. Hear Res 1990;47:191-203.

29. Miettinen S, Laurell G, Andersoson A, Johanson R, Laukikainen $E$. Blood Flow-Independent Accumulation of Cisplatin in the Guinea Pig Cochea. Acta Otolaryngol (Stockh) 1997;117:55-60.

30. Dehne N, Lautermann J, Petrat F, Rauen U, de Groot H. Cisplatin ototoxicity: involvement of iron and enhanced formation of superoxide anion radicals. Toxicol Appl Pharmacol 2001;1174(1):27-34.

31. Watanabe KI, Hess A, Bloch W, Michel O. Nitric oxide synthase inhibitor suppresses the ototoxic side effect of cisplatin in guinea pigs. Anticancer Drugs 2000;11(5):401-6

32. Evans $P$, Halliwell $B$. Free radicals and hearing. Cause consequence and criteria. Ann N Y Acad Sci 1999;28;884:19-40.
33. Huang $T$, Cheng AG, Stupak H, Liu W, Kim A, Staecker $H$, Lefebvre PP, Malgrange B, Kopke R, Moonen G, Van De Water TR. Oxidative stress-induced apoptosis of cochlear sensory cells: otoprotective strategies. Int J Dev Neurosci 2000;18(2-3):259-70.

34. Maheswari KU, Ramachandran T, Rajaji D. Interaction of cisplatin with planar model membranes - dose dependent change in electrical characteristics. Biochim Biophys Acta 2000;15;1463(2):230-40.

35. Rybak LP, Whitworth MA, Somani S. Application of Antioxidants and 0 ther Agents to Prevent Cisplatin Ototoxicity. The Laringoscope Nov 1999;109:1740-4.

36. Cardinaal RM, Groot JCMJ, Huizing EH, Veldman JE, Smoorenburg GF. Histological Effects of Co-administration of an ACTH (4-9) Analog ORG 2766 on Cisplatin Ototoxicity in the Albino Guinea Pig. Hearing Res 2000;144:157-67.

37. Laurell G, Engström B. The Combined Effect of Cisplatin and Furosemida on Hearing Function in Guinea Pigs. Hearing Res 1989;38:19-26

38. Feghali JG, Liu W, Van De Water TR. L-n-acetyl-cysteine protection against cisplatin-induced auditory neuronal and hair cell toxicity. Laryngoscope 2001;111(7):1147-50.

39. Gao WQ. Role of neurotrophins and lectins in prevention of ototoxicity. Ann N Y Acad Sci 1999;28:312-27.

40. Rybak LP, Somani S. Ototoxicity. Amelioration by protective agents Ann N Y Acad Sci. 1999;28:143-51.

41. Cardinaal RM, de Groot JC, Huizing EH, Veldman JE, Smoorenburg GF. Histological effects of co-administration of an ACTH((4-9)) analogue ORG 2766 on cisplatin ototoxicity in the albino guinea pig. Hear Res 2000;144(1-2):157-67.

42. Kamimura T, Whitworth CA, Rybak LP. Effect of 4-methylthiobenzoic acid on cisplatin-induced ototoxicity in the rat. Hear Res 1999;131(12):117-27.

43. Rybak LP, Husain K, Whitworth C, Somani SM. Dose dependent protection by lipoic acid against cisplatin-induced ototoxicity in rats: antioxidant defense system.Toxicol Sci 1999;47(2):195-202.

44. Heijmen PS, Klis SF, De Groot JC, Smoorenburg GF. Cisplatin ototoxicity and the possibly protective effect of alpha-melanocyte stimulating hormone. Hear Res 1999;128(1-2):27-39.

45. Lopez-Gonzalez MA, Guerrero JM, Rojas F, Delgado F. Ototoxicity caused by cisplatin is ameliorated by melatonin and other antioxidants. J Pineal Res 2000;28(2):73-80.

46. Fukaya $H, K a n n o ~ H$. Experimental studies of the protective effect of ginkgo biloba extract (GBE) on cisplatin-induced toxicity in rats. Nippon Jibiinkoka Gakkai Kaiho1999;102(7):907-17.

47. Jordan JA, Schwade ND, Truelson JM. Fosfomycin does not inhibit the tumoricidal efficacy of cisplatinum. Laryngoscope 1999;109(8):1259-62.

48. Canedo DJM. Resistência a Ação Ototóxica de Antibióticos Aminoglicosídeos. Tese de Mestrado. Faculdade de Medicina de Ribeirão Preto - USP. 1999.

49. Cardinaal RM, Groot JCMJ, Huizing EH, Veldman JE, Smoorenburg GF. Cisplatin-Induced Ototoxicity: Morphological Evidence of Spontaneous Outer Harir Cll Recovery in Albino Guinea Pigs? Hearing Res 2000;144147-156.

50. Smoorenburg GF, De Groot JC, Hamers FP, Klis SF. Protection and spontaneous recovery from cisplatin-induced hearing loss. Ann N Y Acad Sci 1999;28;884:192-210.

51. Klis SF, O'Leary SJ, Hamers FP, De Groot JC, Smoorenburg GF. Reversible cisplatin ototoxicity in the albino guinea pig. Neuroreport 2000;11(3):623-6.

52. Ekborn A, Laurell G, Anderson A, Wallim I, Eksborg S, Ehrsson H. Cisplatin-Induced Hearing Loss: influence of the Mode of Drug Administration in the Guinea Pig. Hearing Res 2000;140:38-34.

53. Taudy M, Syka J, Popelár J, Úlehlová L, Carboplatin and Cisplatin Ototoxicity in Guinea Pigs. Audiology 1992;31:293-299.

54. Hatzopoulos S, Di Stefano M, Albertin A, Martini A. Evaluation of cisplatin ototoxicity in a rat animal model. Ann N Y Acad Sci $1999 ; 28: 211-25$ 
55. Saito $T$, Manabe $Y$, Honda $N, Y$ amada $T, Y$ amamoto $T$, Saito $H$. Semiquantitative analysis by scanning electron microscopy of cochlear hair cell damage by ototoxic drugs. Scanning Microsc 1995;9(1):271-80; discussion 280-1.

56. Bridi $R$, Crossetti FP, Steffen VM, Henriques AT. The antioxidant activity of standardized extract of Ginkgo biloba (EGb 761) in rats. Phytother Res 2001;15(5):449-51.

57. Sasaki K, Hatta S, Wada K, Ueda N, Y oshimura T, Endo T, Sakata M, Tanaka T, Haga M. Effects of extract of Ginkgo biloba leaves and its constituents on carcinogen-metabolizing enzyme activities and glutathione levels in mouse liver. Life Sci 2002;22;70(14):1657-67.

58. Carini M, Aldini G, Rossoni G, Morazzoni P, Facino RM. Complexation of Ginkgo biloba extract with phosphatidylcholine improves cardioprotective activity and increases the plasma antioxidant capacity in the rat. Planta Med 2001;67(4):326-30.
59. Siddique MS, Eddeb F, Mantle D, Mendelow AD. Extracts of Ginkgo biloba and Panax ginseng protect brain proteins from free radical induced oxidative damage in vitro. Acta Neurochir Suppl 2000;76:87-90.

60. Louajri A, Harraga S, Godot V, Toubin G, Kantelip JP, Magnin P. The effect of ginkgo biloba extract on free radical production in hypoxic rats. Biol Pharm Bull 2001;24(6):710-2.

61. Gohil K, Moy RK, Farzin S, Maguire JJ, Packer L. mRNA expression profile of a human cancer cell line in response to Ginkgo biloba extract: induction of antioxidant response and the Golgi system. Free Radic Res 2000;33(6):831-49.

62. Fosslien E. Mitochondrial medicine-molecular pathology of defective oxidative phosphorylation. Ann Clin Lab Sci 2001;31(1):25-67. Review. 